\title{
High Field Strength Magnetic Resonance Imaging
}

National Cancer Institute

\section{Source}

National Cancer Institute. High Field Strength Magnetic Resonance Imaging. NCI

Thesaurus. Code C116455.

A type of magnetic resonance imaging that uses larger, stronger magnets to generate faster scans and higher resolution images. 